score $(p<0.01)$, decreased plasma levels of CXCL1 $(p<0.05)$ and reduced gene expression of pro-inflammatory markers (IL-6, TNF-a, IL-1b, INF-g, IL-8; $\mathrm{p}<0.05)$ compared to vehicle. SM04755 treatment promoted tendon regeneration measured as increased expression of tenocyte markers $(p<0.05)$, increased Type I/Type III collagen ratio (Figure $B ; p<0.01$ ) and Sirus Red stained collagen fibers in tendon compared to vehicle. SM04755 treatment increased \% total weight bearing on the affected limb $(p<0.01)$, at multiple time points (Figure $C)$, indicating reduced pain in the rodent model.

Figure. SM04755 inhibited inflammation, promoted tendon healing and reduced pain in a rat collagenase-induced tendinopathy model
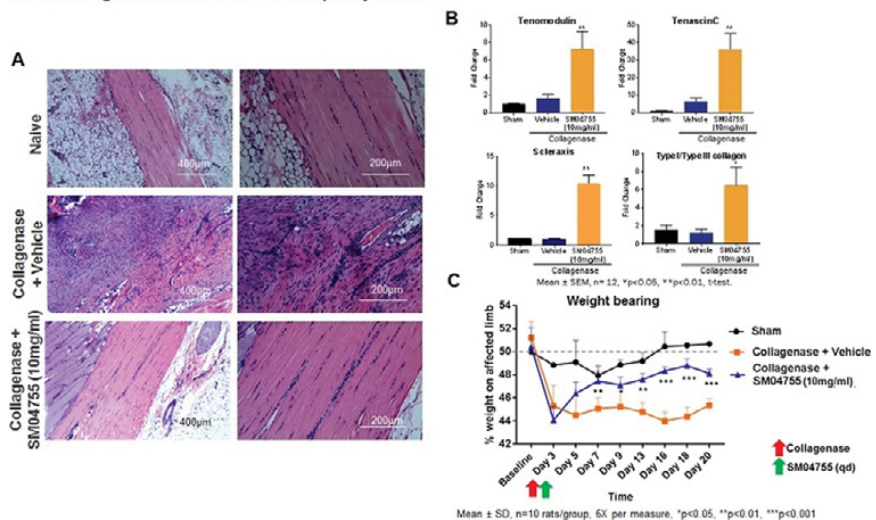

Conclusions: Topical SM04755, a Wnt pathway inhibitor, reduced inflammation, promoted tendon regeneration and healing, and reduced pain compared to vehicle in rodent tendinopathy models. SM04755 is a potential treatment for tendinopathy. Clinical studies are in progress.

Disclosure of Interest: V. Deshmukh Shareholder of: Samumed, LLC, Employee of: Samumed, LLC, T. Seo Shareholder of: Samumed, LLC, Employee of: Samumed, LLC, M. Ibanez Shareholder of: Samumed, LLC, Employee of: Samumed, LLC, J. Stewart Shareholder of: Samumed, LLC, Employee of: Samumed, LLC, B. Hofilena Shareholder of: Samumed, LLC, Employee of: Samumed, LLC, Y. Yazici Shareholder of: Samumed, LLC, Employee of: Samumed, LLC DOI: 10.1136/annrheumdis-2017-eular.6427

\section{SAT0608 RELATION BETWEEN SCAPULAR MUSCLE ENDURANCE, MUSCLE STRENGTH, PAIN AND FUNCTION IN PATIENTS WITH ROTATOR CUFF LESION}

U. Sozlu, S. Basar, U. Kanatli, Z.B. Alkan. Gazi University, Ankara, Turkey

Background: Rotator cuff lesion (RCL) is an term that encompasses a spectrum of shoulder conditions including; subacromial impingement syndrome (SIS), partial and full thickness rotator cuff tears (RCT) and calcific tendinitis. Symptoms include pain, limited motion, muscle weakness and functional disability.

Objectives: To investigate the relationship between scapular muscle endurance (SME), muscle strength, pain and function in patients with shoulder RCL.

Methods: This study was conducted on 53 patients in shoulder RCL and 23 healthy controls. SIS was present in 25 patients $(14$ males, 11 females; mean age $41 \pm 9$ years) and partial RCT in 28 patients ( 16 females, 12 males; mean age was $54 \pm 5$ years) in the study group. In the control group, there were 12 females and 11 males and the mean age was $48 \pm 10$ years. SME was measured as isometric based on the exercise developed by Sahrmann. Strength of the shoulder and scapular muscles were evaluated with digital manuel muscle dynamometer. Activity, night and rest pain levels were evaluated by visual analog scale. Functional activity status was assessed by the Functional Impairment Test-Head, and Neck/Shoulder/Arm (FIT-HaNSA).

Results: Statistically significant relationship was found positively in varying grades between SME and serratus anterior, middle trapezius, shoulder flexor, extensor, abductor, supraspinatus, pectoralis majör strength in partial RCT group and between lower trapezius muscle strength and SME in addition to these muscles in SIS group $(p<0.05)$. Statistically significant relationship at varying degrees was found between SME and all muscles excluding shoulder internal, external rotators in control group $(p<0.05)$. In SIS and partial RCT groups, negative, moderate and statistically significant relationship was found between SME and activity pain $(p<0.05)$. In SIS and partial RCT groups significant correlation was not observed between SME and rest, night pain levels $(p>0.05)$. In SIS and partial RCT groups, positive, excellent and statistically significant relationship was detected between SME and FIT-HaNSA $(p<0.05)$. In control group, positive, moderate, again statistically significant relationship was detected $(p<0.05)$.

Conclusions: Muscle strength may be reduced, upper extremity function may worsen and activity pain may increase in patients with reduced SME. It was concluded that shaping of exercise programs of muscle groups related to SME in rehabilitation studies and SME should be increased in patients with SIS and partial RCT in order to reduce shoulder pain and improve its functionality.

References:

[1] Cofield, R. H. (1985). Current concepts review rotator cuff disease of the shoulder. Journal of Bone and Joint Surgery. American volume, 67(5), 974-979.

[2] Neer, C. S. (1993). Impingement lesions. Clinical Orthopedia, 173, 71-7.

[3] Edmondston, S. J., Wallumrød, M. E., MacLéid, F., Kvamme, L. S., Joebges, S. and Brabham, G. C. (2008). Reliability of isometric muscle endurance tests in subjects with postural neck pain. Journal of Manipulative and Physiological Therapeutics, 31(5), 348-354.

[4] MacDermid, J. C., Ghobrial, M., Quirion, K. B., St-Amour, M., Tsui, T., Humphreys, D., McCluskie, J., Shewayhat, E. and Galea, V. (2007). Validation of a new test that assesses functional performance of the upper extremity and neck (FIT-HaNSA) in patients with shoulder pathology. BMC Musculoskeletal Disorders, 8(1), 42.

Disclosure of Interest: None declared

DOI: 10.1136/annrheumdis-2017-eular.6310

\section{SATURDAY, 17 JUNE 2017 Diagnostics and imaging procedures}

\section{SAT0609 GIANT CELL ARTERITIS: ATHEROSCLEROSIS AS PITFALL IN THE HALO SIGN}

E. De Miguel ${ }^{1}$, L.M. Beltran ${ }^{2}$, F. Deodati ${ }^{2}$, I. Monjo ${ }^{1}$, W.A. Schmidt ${ }^{3}$,

J. García-Puig ${ }^{2} .{ }^{1}$ Rheumatology; ${ }^{2}$ Internal Medicine, Hospital Universitario la Paz, Madrid, Spain; ${ }^{3}$ Rheumatology, Immanuel Krankenhaus, Berlin, Germany

Background: The halo sign (defined as an homogenous, hypoechoic wall thickening, well delineated towards the luminal side, visible in longitudinal and transverse planes). is the more accurate ultrasonographic (US) finding for the diagnosis of Giant Cell Arteritis (GCA). False-positive halos are seen in malignant and infectious diseases. The new US high frequencies probes make possible not only to see the halo sign but also measure the increase of the intima-mediathickness (IMT). Moreover, the US appearance of the IMT in atherosclerotic carotid artery may be hypoechoic as the halo sign.

Objectives: The aim of this study was to explore if the increase of the atherosclerotic IMT can mimic the halo sign in temporal arteries (TA) and to produce false positives in the diagnosis of GCA.

Methods: Consecutive non selected patients, $>50$ years-old with high vascular risk according to European Guidelines on Cardiovascular Disease Prevention, and without signs or symptoms of GCA, were included.

Ultrasonography of carotid artery: Carotid ultrasound examinations were performed on a Mylab Seven (Esaote Medical Systems, Italy) with a 4-13 MHz linear-array. The system employed dedicated software radiofrequency-tracking technology to obtain IMT $\left(\mathrm{QIMT}^{\circledR}\right)$.

Ultrasonography of temporal superficial artery: A color Doppler ultrasound (CDU) and grey scale measure of the IMT/halo sign in the branches of both TA was performed by a second experienced sonographer. A Mylab Twice equipment (Esaote, Geneve, Italy) was used, with a $22 \mathrm{MHz}$ frequency for grey scale and a $12.5 \mathrm{MHz}$ for CDU, with a color gain of 51 and a PRF of $2 \mathrm{kHz}$. The sonographer was blind to the clinical data and carotid ultrasound IMT measures. Examination videos were stored for reliability and an intra-reader was performed months after the examination.

Statistical analysis: Descriptive frequencies, Student's $t$-test, Cronbach's alpha and Spearman correlations was used.

Results: Forty patients were studied, 28 men $(70 \%)$, with a mean age of $70,6 \pm 6,9$ years. Three patients were active smoker and 27 ex-smokers. Arterial hypertension was present in $39(97.5 \%)$, dyslipidemia in $34(85 \%)$ and diabetes in $19(47.5 \%)$. The mean erythrocyte sedimentation rate was $13.6 \pm 11.0$. Eighty carotids were studied, 50 had plaques and 30 did not with a IMT ranged from 0.528 to $1.480 \mathrm{~mm}$. The mean values of TA related with the carotid IMT are presented in the table: the increase in the carotid IMT is associated with an increase in the IMT of the TA with a weak Spearman correlation (parietal branches $0.282 p=0.012$ and frontal branches $0.228 p=0.048$ )

Patients with a final GCA diagnosis $(n=22)$

\begin{tabular}{lcccc}
\hline Carotid IMT mm & \multicolumn{4}{c}{ Branches of Temporal arteries parietal and frontal } \\
\cline { 2 - 5 } & $\mathrm{n}$ & Mean Halo/IMT mm & SD & Min-Max \\
\hline$\leq 0.7$ & 12 & 0.247 & \pm 0.043 & $0.16-0.29$ \\
$\leq 0.9$ & 46 & 0.254 & \pm 0.037 & $0.18-0.33$ \\
$\leq 1$ & 42 & 0.269 & \pm 0.034 & $0.18-0.36$ \\
$\leq 1.2$ & 50 & 0.282 & \pm 0.052 & $0.17-0.45$ \\
$\geq 1.2$ & 10 & 0.284 & \pm 0.067 & $0.19-0.40$ \\
\hline
\end{tabular}

From reliability a Cronbach's alpha of 0.900 and 0.876 were achieved for parietal and frontal branches respectively.

Some patients had a TA IMT that can be interpreted as halo sign: $18(45 \%)$ patients and $33(20.6 \%)$ TA branches if we choose a cutoff $>0.30 \mathrm{~mm}$ of IMT/halo sign; $4(10 \%)$ patients and $7(4.4 \%)$ TA branches if the cutoff were $>0.34 \mathrm{~mm}$.

Conclusions: The atherosclerotic disease can produce false-positive GCA diagnosis. Carotid IMT $>0.9 \mathrm{~mm}$ is associated with halo sign in TA. The IMT cutoff value for the diagnosis of GCA should be established.

Disclosure of Interest: None declared

DOI: 10.1136/annrheumdis-2017-eular.1461 